\title{
Stimulus affect valence may influence mapping-rule selection but does not reverse the spatial compatibility effect: reinterpretation of Conde et al. (2011)
}

\author{
Robert W. Proctor \\ Purdue University, West Lafayette, IN, USA
}

\begin{abstract}
Conde et al. (2011) reported a finding that their article title characterized as "stimulus affective valence reverses the spatial compatibility effect." In their study, participants performed a choice-reaction task in which the stimulus was a soccer player from their "Favorite" team or from a "Rival" team, presented in a left or right location. The team signaled whether a spatially compatible or incompatible keypress was to be made in response to the stimulus location. The Favorite team showed a benefit for the spatially compatible response, but the Rival team showed a benefit for the spatially incompatible response. In the present commentary, the data of Conde et al. are reorganized according to the two mixed-mapping conditions under which participants performed: Favorite $\rightarrow$ compatible/Rival $\rightarrow$ incompatible and Rival $\rightarrow$ compatible/Favorite $\rightarrow$ incompatible. This reorganization shows the typical finding of no spatial compatibility effect for mixed mappings in both conditions but an overall advantage for the Favorite $\rightarrow$ compatible/Rival $\rightarrow$ incompatible mapping of teams to mapping rules. This compatibility effect for team preference to mapping rule may be a consequence of positive and negative affect, although other accounts are possible. Regardless of its basis, that compatibility effect did not modulate the spatial compatibility effect. Keywords: mapping selection, mixed-mappings, stimulus-response compatibility.
\end{abstract}

Received 4 November 2012; received in revised form 3 January 2013; accepted 2 February 2013. Available online 27 June 2013.

One of the most robust phenomena in human performance is the spatial stimulus-response compatibility (SRC) effect: Reaction time (RT) is shorter when the mapping of stimulus locations to response locations is spatially compatible than when it is not (Fitts \& Deininger, 1954; see Proctor \& Vu, 2006, for a review). For two-choice reaction tasks, mean RT is characteristically at least $50 \mathrm{~ms}$ shorter with a compatible mapping of left and right stimulus locations to left and right keypresses made with the index fingers of each hand than with an incompatible mapping (e.g., Brebner, Shepard, \& Cairney, 1972). Because this spatial SRC effect occurs even when the hands are crossed such that the left key is operated with the right index finger and the right key with the left index finger and depends on the relative positions of the alternative stimuli and responses, it is typically attributed to the processes involved in relating stimulus and response spatial codes (e.g., Anzola, Bertoloni, Buchtel, \& Rizzolatti, 1977; Umiltà \& Nicoletti, 1990).

Robert W. Proctor, Department of Psychological Sciences, Purdue University. Correspondence concerning this paper should be addressed to Robert W. Proctor, Department of Psychological Sciences, Purdue University, 703 Third St., West Lafayette, IN 47907-2081. E-mail: proctor@psych. purdue.edu

\section{Conde et al. (2011) Study}

Recently, Conde et al. (2011) published an article in which they interpreted the results as showing reversal of the two-choice spatial SRC effect when the stimuli had negative affective valence. Their procedure was as follows. Participants from Brazil first rank ordered their preferences for the four major soccer teams of Rio de Janeiro. The first and fourth teams for each individual participant were designated as Favorite and Rival teams, and then figures of players dressed in the uniforms of the two teams were used as stimuli in a choice-reaction task. For the task, a figure was presented in a left or right location, and whether to respond compatibly or incompatibly to the stimulus location was designated by the soccer team. The spatially compatible keypress was to be made if the figure was from one team and the spatially incompatible keypress if the figure was from the other team. Each participant performed two blocks of 120 trials, counterbalanced for order, one for which the Favorite team signaled a spatially compatible mapping rule and the Rival team an incompatible mapping rule, and the other for which the Rival team signaled a spatially compatible mapping rule and the Favorite team an incompatible mapping rule.

The mean RTs were analyzed as a function of Preference (Favorite vs. Rival), Hemifield (Left vs. Right), and Response Key (Left vs. Right). The only 

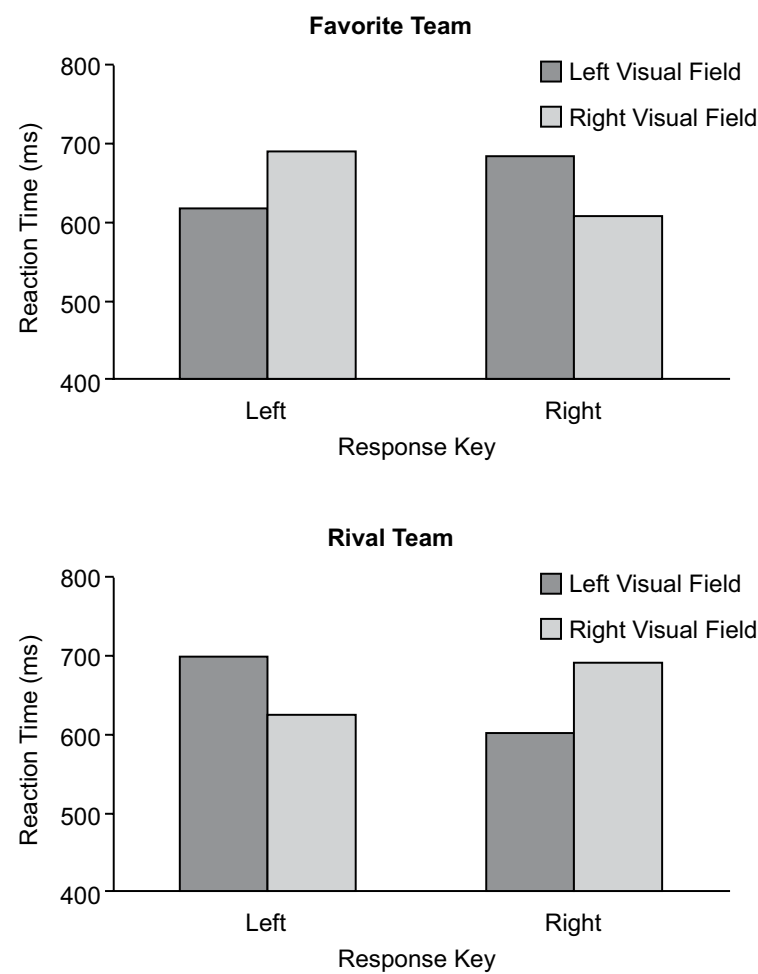

Figure 1. Mean reaction time, as plotted and analyzed by Conde et al. (2011), shown for Favorite and Rival teams, as a function of Visual Field (Left, Right), and Response Key (Left, Right).

significant effect yielded by the analysis of variance (ANOVA) was a three-way interaction of Preference, Hemifield, and Response Key, $F(1,13)=6.601, p=$ .023. Conde et al. (2011) reported follow-up analyses separately for the Favorite team (Figure 1, top panel) and the Rival team (Figure 1, bottom panel), with the former showing a 73-ms benefit for the compatible mapping and the latter an opposite 81-ms benefit for the incompatible mapping. That is, the Rival team showed a reversed spatial compatibility effect, which Conde et al. interpreted as evidence for modulation of spatial compatibility that "may result from approach/ avoidance reactions to the Favorite and Rival Teams, respectively" (p. 81). In other words, their explanation was that the incompatible response from the Rival team was preferred over the compatible response because the former was "away" from the stimulus.

\section{Reorganization and Reinterpretation of the results of Conde et al. (2011)}

The manipulation of Favorite and Rival teams in the study of Conde et al. (2011) is clever and does show an influence of that distinction on performance, but there is a simpler interpretation of their results for which that variable does not modulate spatial SRC, contrary to their explanation. The experiment of Conde et al. is a variant of a mixed-mapping task in which participants are to make the spatially compatible response on some trials and the spatially incompatible response on others. The influence of mixed mappings was first investigated by Shaffer (1965) who found a nonsignificant $-8 \mathrm{~ms}$
SRC effect (i.e., slightly shorter RT for the incompatible mapping than for the compatible mapping) when the mapping was signaled by a horizontal or vertical line presented simultaneously with the left and right stimulus. This outcome compared to a 54-ms SRC effect obtained when the compatible and incompatible mappings were presented in distinct trial blocks. This result has since been replicated in several studies using line orientation (Proctor, Yamaguchi, Dutt, \& Gonzalez, 2012) or color of the left or right stimulus as the mapping signal (Heister \& Schroeder-Heister, 1994; Vu \& Proctor, 2004, 2008, 2011; Yamaguchi \& Proctor, 2006). When compatible and incompatible mappings are mixed, the compatible mapping predictably shows no benefit over the incompatible mapping and often a tendency toward a slight cost.

By performing their follow-up analyses separately for the Favorite and Rival teams, half of the data points within each of the analyses of Conde et al. (2011) came from the trial block for which the Favorite team signaled the spatially compatible mapping rule and the Rival team the incompatible mapping rule and half from the trial block for which the respective teams signaled the opposite mapping rules. For example, for the Favorite team, the means for compatible locations (left stimulus $\rightarrow$ left response; right stimulus $\rightarrow$ right response) came from the trial block for which the mapping was Favorite $\rightarrow$ compatible/Rival $\rightarrow$ incompatible, whereas the means for the incompatible locations (left stimulus $\rightarrow$ right response; right stimulus $\rightarrow$ left response) came from the trial block for which the mapping was Rival $\rightarrow$ compatible/ Favorite $\rightarrow$ incompatible. Because the alternative mappings of teams to rules in the different trial blocks may themselves not be equivalent, a more appropriate way to follow up the three-way interaction is with comparisons of the compatibility effects within each of the trial blocks for the respective mappings of teams to rules.

When the means presented by Conde et al. are reorganized in this manner (see Figure 2), the following emerges. For the trial block in which the Favorite team signaled the compatible rule and the Rival team the incompatible rule (Figure 2, top panel), the mean RTs for the two signals were 613.5 and $614 \mathrm{~ms}$, respectively. Similarly, for the trial block in which the Rival team signaled compatible and the Favorite team signaled incompatible (Figure 2, bottom panel), the mean RTs were 695 and $686.5 \mathrm{~ms}$. In neither of the mixed-mapping trial blocks was there a spatial SRC effect, in agreement with the results obtained in the previously cited mixedmapping studies by Shaffer (1965) and others.

This reorganization of the data of Conde et al. (2011) shows little evidence that the spatial compatibility effect is modulated by whether the Favorite or Rival team signals the compatible or incompatible mapping rule. Therefore, what accounts for the significant three-way interaction in their main ANOVA? In that ANOVA, Preference was used as the third independent variable, in addition to Hemifield and Response Key, and thus the Favorite and Rival conditions included data from the different mapping- 

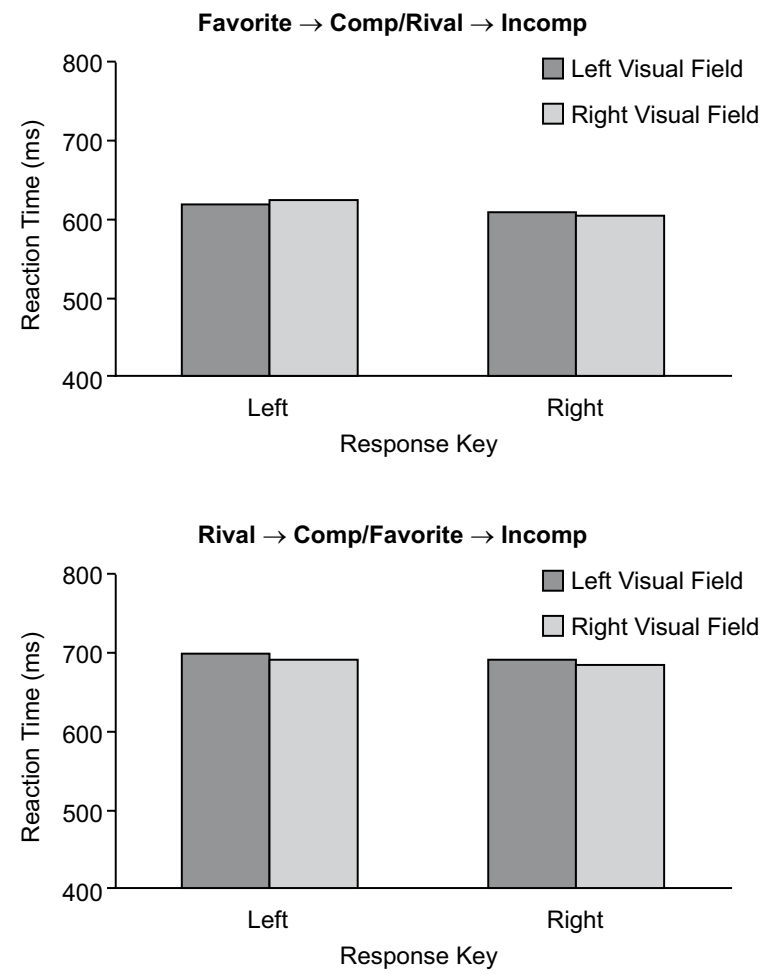

Figure 2. Mean reaction-time data from Figure 1 re-plotted separately for each Mapping Condition, as a function of Visual Field (Left, Right), and Response Key (Left, Right). Comp = compatible; Incomp $=$ incompatible

rule blocks as in the follow-up two-way ANOVAs. If the data were reanalyzed with Mapping-Rule Block as the variable, as in Figure 2, the $F$ ratios would remain the same (because the same numbers are used), but they would be rearranged because the individual conditions are divided up differently. In this ANOVA, the significant term would shift to be the main effect of Mapping-Rule Block as is evident in Figure 2, where RT was $614 \mathrm{~ms}$ when the Favorite team signaled the compatible mapping rule and the Rival team the incompatible mapping rule, compared to $691 \mathrm{~ms}$ when the Rival team signaled the compatible mapping rule and the Favorite team the incompatible mapping rule. In other words, participants were able to select which mapping rule to apply faster when the compatible rule was signaled by the Favorite team and the incompatible rule by the Rival team than with the opposite mapping. This compatibility effect for the dimension of team preference to mapping rule is an intriguing result, but it has no significant impact on spatial compatibility. To emphasize, the three-way ANOVA reported by Conde et al. showed only one significant effect which, when the data are analyzed as in Figure 2, is the main effect of the Mapping-Rule Block. That no other $F$ ratio was significant indicates that there was no spatial SRC effect overall and no interaction with Mapping-Rule Block as evident in Figure 2.

\section{Discussion}

The compatibility effect between team preference and mapping rules is of interest because there is no obvious overlap or similarity between the two dimensions (Kornblum, 1992). One possible basis of the effect is the positive and negative affect associated with the stimulus dimension as Conde et al. (2011) proposed. If compatible and incompatible mappings also have positive and negative affect, respectively, then there would be overlap along stimulus and mappingrule affect dimensions, yielding a compatibility effect (e.g., Horstman, 2010). A more likely alternative is that the Favorite team is coded as "compatible" and the Rival team as "incompatible," and it is easier to select the appropriate mapping rule when the compatible team is mapped to the compatible rule and the incompatible team to the compatible rule than with the opposite mapping. Such a result would be consistent with evidence that explicit selection between mapping rules is a distinct stage when task mappings are mixed (e.g., Proctor \& Vu, 2009).

Still another possibility is that the teams are not coded as compatible and incompatible, and thus there is no conceptual overlap with the mapping-rule distinction. However, the members of each dimension are coded asymmetrically as in linguistic marking, with one member being dominant, or unmarked, and the other being "marked" relative to it (e.g., Nuerk, Iversen, \& Willmes, 2004), resulting in codes of different polarities for the members of the two dimensions. If Favorite is coded as the unmarked (or "+" polarity) member of the dimension of preference and Rival as marked relative to it (or "-" polarity) and compatible is coded as unmarked ("+" polarity) for the dimension of compatibility and incompatible as marked ("--" polarity), this correspondence of the polarities could produce the observed compatibility effect of team preference with mapping rules without any overlap of conceptual content between the dimensions (Proctor \& Cho, 2006).

In summary, the ingenious study of Conde et al. (2011) demonstrates a large compatibility effect for the mapping of team preference to selection of compatible versus incompatible spatial mapping rule. Although this compatibility effect may have its basis in affective coding of the teams, other accounts that do not invoke affect are possible. Regardless of the basis for the influence of team preference on selection of the spatially compatible or incompatible mapping rule, it did not modulate the spatial compatibility effect, which is typically absent when mappings are mixed. Hence, there is no reason to postulate approach-avoidance tendencies as a factor in the study of Conde et al. A more general point illustrated by this reorganization of their data is that caution should be exercised when comparing results across trial blocks with different stimulus-response mappings.

\section{References}

Anzola, G. P., Bertoloni, G. G., Buchtel, H. A., \& Rizzolatti, G. G. (1977). Spatial compatibility and anatomical factors in simple and choice reaction time. Neuropsychologia, 15, 295-302.

Brebner, J., Shepard, M., \& Cairney, P. (1972). Spatial relationships and S-R compatibility. Acta Psychologica, 36, 1-15. 
Conde, E. F. Q., Jazenko, F., Filho, R. S. F., da Costa, D. H., TorroAlves, N., Cavallet, M., \& Gawryszewski, L. G. (2011). Stimulus affective valence reverses spatial compatibility effect. Psychology \& Neuroscience, 4, 81-87.

Fitts, P. M., \& Deininger, R. L. (1954). S-R compatibility: Correspondence among paired elements within stimulus and response codes. Journal of Experimental Psychology, 48, 483-492.

Heister, G., \& Schroeder-Heister, P. (1994). Spatial S-R compatibility: Positional instructions vs. compatibility instruction. Acta Psychologica, 85, 15-24.

Horstmann, G. (2010). Tone-affect compatibility with affective stimuli and affective responses. Quarterly Journal of Experimental Psychology, 63, 2239-2250.

Kornblum, S. (1992). Dimensional overlap and dimensional relevance in stimulus-response and stimulus-stimulus compatibility. In G. E. Stelmach \& J. Requin (Eds.), Tutorials in motor behavior (Vol. 2, pp. 743-777). Amsterdam: North-Holland.

Nuerk, H.-C., Iversen, W., \& Willmes, K. (2004). Notational modulation of the SNARC and the MARC (linguistic markedness of response codes) effect. Quarterly Journal of Experimental Psychology, 57, 835-863.

Proctor, R. W., \& Cho, Y. (2006). Polarity correspondence: A general principle for performance of speeded binary classification tasks. Psychological Bulletin, 132, 416-442.

Proctor, R. W., \& Vu, K.-P. L. (2006). Stimulus-response compatibility principles: Data, theory, and application. Boca Raton, FL: CRC Press.
Proctor, R. W., \& Vu, K.-P. L. (2009). Determinants of the benefit for consistent spatial mappings in dual-task performance of three-choice tasks. Attention, Perception, \& Psychophysics, 71, 1771-1781.

Proctor, R. W., Yamaguchi, M., Dutt, V., \& Gonzalez, C. (2012). Dissociation of S-R compatibility and Simon effects with mixed tasks and mappings. Journal of Experimental Psychology: Human Perception and Performance, 39, 593-609.

Shaffer, L. H. (1965). Choice reaction with variable S-R mapping. Journal of Experimental Psychology, 70, 284-288.

Umiltá, C., \& Nicoletti, R. (1990). Spatial stimulus-response compatibility. In R. W. Proctor \& T. Reeve (Eds.), Stimulusresponse compatibility: An integrated perspective (pp. 89-116). Amsterdam: North-Holland.

Vu, K.-P. L., \& Proctor, R. W. (2004). Mixing compatible and incompatible mappings: Elimination, reduction, and enhancement of spatial compatibility effects. Quarterly Journal of Experimental Psychology, 57A, 539-556.

Vu, K.-P. L., \& Proctor, R. W. (2008). Age differences in response selection for pure and mixed stimulus-response mappings and tasks. Acta Psychologica, 129, 49-60.

Vu, K.-P. L., \& Proctor, R. W. (2011). Influence of bias on visuospatial and verbal compatibility effects in mixed mapping environments. Attention, Perception, \& Psychophysics, 73, 2594-2605.

Yamaguchi, M., \& Proctor, R. W. (2006). Stimulus-response compatibility with pure and mixed mappings in a flight task environment. Journal of Experimental Psychology: Applied, 12, 207-222. 